\title{
Challenging shock models with SOFIA OH observations in the high-mass star-forming region Cepheus $A$
}

\author{
A. Gusdorf ${ }^{1,2}$, R. Güsten ${ }^{3}$, K. M. Menten ${ }^{3}$, D. R. Flower ${ }^{4}$, G. Pineau des Forêts ${ }^{5}$, C. Codella ${ }^{6}$, T. Csengeri ${ }^{3}$, \\ A. I. Gómez-Ruiz ${ }^{3}$, S. Heyminck ${ }^{3}$, K. Jacobs ${ }^{7}$, L. E. Kristensen ${ }^{8}$, S. Leurini ${ }^{3}$, M. A. Requena-Torres ${ }^{3}$, \\ S. F. Wampfler ${ }^{9}$, H. Wiesemeyer ${ }^{3}$, and F. Wyrowski ${ }^{3}$
}

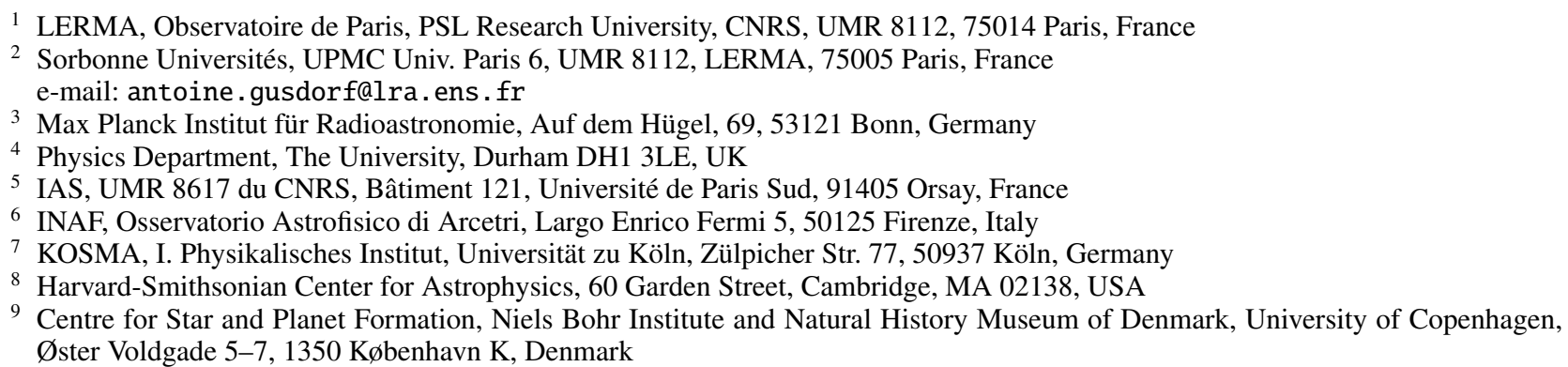

Received 12 November 2014 / Accepted 26 August 2015

\section{ABSTRACT}

\begin{abstract}
Context. $\mathrm{OH}$ is a key molecule in $\mathrm{H}_{2} \mathrm{O}$ chemistry, a valuable tool for probing physical conditions, and an important contributor to the cooling of shock regions around high-mass protostars. OH participates in the re-distribution of energy from the protostar towards the surrounding Interstellar Medium.

Aims. Our aim is to assess the origin of the $\mathrm{OH}$ emission from the Cepheus A massive star-forming region and to constrain the physical conditions prevailing in the emitting gas. We thus want to probe the processes at work during the formation of massive stars. Methods. We present spectrally resolved observations of $\mathrm{OH}$ towards the protostellar outflows region of Cepheus A with the GREAT spectrometer onboard the Stratospheric Observatory for Infrared Astronomy (SOFIA) telescope. Three triplets were observed at 1834.7 GHz, $1837.8 \mathrm{GHz}$, and $2514.3 \mathrm{GHz}\left(163.4 \mu \mathrm{m}, 163.1 \mu \mathrm{m}\right.$ between the ${ }^{2} \Pi_{1 / 2} J=3 / 2$ and $J=1 / 2$ states, and $119.2 \mu \mathrm{m}$, a ground transition between the ${ }^{2} \Pi_{3 / 2} J=5 / 2$ and $J=3 / 2$ states), at angular resolutions of $16^{\prime \prime} 3,16^{\prime \prime} 3$, and $11^{\prime \prime} .9$, respectively. We also present the $\mathrm{CO}(16-15)$ spectrum at the same position. We compared the integrated intensities in the redshifted wings to the results of shock models.

Results. The two $\mathrm{OH}$ triplets near $163 \mu \mathrm{m}$ are detected in emission, but with blending hyperfine structure unresolved. Their profiles and that of CO (16-15) can be fitted by a combination of two or three Gaussians. The observed $119.2 \mu \mathrm{m}$ triplet is seen in absorption, since its blending hyperfine structure is unresolved, but with three line-of-sight components and a blueshifted emission wing consistent with that of the other lines. The $\mathrm{OH}$ line wings are similar to those of $\mathrm{CO}$, suggesting that they emanate from the same shocked structure.

Conclusions. Under this common origin assumption, the observations fall within the model predictions and within the range of use of our model only if we consider that four shock structures are caught in our beam. Overall, our comparisons suggest that all the observations might be consistently fitted by a J-type shock model with a high pre-shock density $\left(n_{\mathrm{H}}>10^{5} \mathrm{~cm}^{-3}\right)$, a high shock velocity $\left(v_{\mathrm{s}} \gtrsim 25 \mathrm{~km} \mathrm{~s}^{-1}\right)$, and with a filling factor of the order of unity. Such a high pre-shock density is generally found in shocks associated to high-mass protostars, contrary to low-mass ones.
\end{abstract}

Key words. astrochemistry - stars: formation - ISM: jets and outflows - ISM: kinematics and dynamics - infrared: ISM ISM: individual objects: Cep A

\section{Introduction}

Observations over the past few decades have shown that, in the early stages of star formation, the process of mass accretion is almost always associated with mass ejection in the form of collimated jets. The jets impact on the parent cloud, driving a shock front through the collapsing interstellar gas. Large cavities, called bipolar outflows, are carved in the ambient medium, which is accelerated, compressed and heated by the shock wave. This paradigm was proposed a few decades ago by Snell et al. (1980), in connection with the formation of low-mass stars, and has been regularly verified in such environments (see, for example, Arce et al. 2007; Frank et al. 2014, for reviews), including recent high-angular-resolution observations by ALMA (e.g. Codella et al. 2014). However, establishing its applicability to the formation of massive stars remains a challenge for observers and modellers (see Tan et al. 2014, for a review of massive star formation). A central question is whether the shocks that are generated by massive protostars have similar physical and chemical properties to those driven by young stellar objects (YSOs) of lower mass. Studying the molecular emission from star-forming regions (SFRs) is a way to progress on this question. 
In non-dissociative shock waves, the kinetic temperature of the gas can rise to a few thousand degrees, at which point the energy barriers to numerous chemical reactions can be overcome. Other processes affect the dust grains, resulting in a significant alteration of the abundances of certain species (Bachiller et al. 2001; Flower \& Pineau des Forêts 2003). Among the shocktracing molecules, water is particularly important: it is a carrier of oxygen, a relatively abundant element that modifies the gasphase or grain-surface chemistry of many other species, and is an important coolant of the gas (see, for example, van Dishoeck et al. 2011, 2013, 2014, for reviews of these aspects). If its chemistry were properly understood, $\mathrm{H}_{2} \mathrm{O}$ would be a most appropriate molecule to comparatively study the formation of stars of various masses. However, this is not the case, and, with a view to understanding the abundance of $\mathrm{H}_{2} \mathrm{O}$ and to characterizing the nature of the shock waves generated during the star formation process, the hydroxyl radical has emerged as a key species (see Wampfler et al. 2013, hereafter W13, for an overview of previous studies of $\mathrm{OH}$ ). $\mathrm{OH}$ is chemically linked to $\mathrm{H}_{2} \mathrm{O}$ through the $\mathrm{OH}+\mathrm{H}_{2} \Longleftrightarrow \mathrm{H}_{2} \mathrm{O}+\mathrm{H}$ reactions. The formation of $\mathrm{H}_{2} \mathrm{O}$ from $\mathrm{OH}$ is expected to be efficient in jets and outflows through both high-temperature gas-phase and grain-surface chemistry. Below around $250 \mathrm{~K}$, "standard" gas-phase chemistry applies, in which $\mathrm{H}_{2} \mathrm{O}$ is formed and destroyed principally through ion-molecule reactions.

$\mathrm{OH}$ is a product of $\mathrm{H}_{2} \mathrm{O}$ photodissociation, and some observational studies have linked a lack of $\mathrm{H}_{2} \mathrm{O}$ directly to an enhanced abundance of $\mathrm{OH}$. This photodissociation may be driven by the radiation field of the protostar, in low-mass YSOs (as mentioned in Wampfler et al. 2010; Karska et al. 2013, 2014b) and high-mass YSOs (as evoked in Karska et al. 2014a and Wampfler et al. 2011, hereafter W11). It might also be driven by the radiation emitted by the shock wave itself in low-mass YSOs (as mentioned in Wampfler et al. 2010; Karska et al. 2014b). For instance, in the bipolar outflow system HH 211, driven by a lowmass YSO, Tappe et al. $(2008,2012)$ have attributed the detection of superthermal $\mathrm{OH}$ emission (i.e. resulting from the population of rotational levels up to at least $28200 \mathrm{~K}$ despite the high $A$ coefficeint values) to the photodissociation of $\mathrm{H}_{2} \mathrm{O}$ by the UV radiation generated in the terminal shock. The photodissociation may also be triggered by an external source of radiation, such as an intermediate-mass YSO irradiating lower mass SFRs (Lindberg et al. 2014). Alternatively, $\mathrm{OH}$ has been proposed as a tracer of dissociative shocks (Flower \& Pineau des Forêts 2013) around both low-mass (Wampfler et al. 2010; Benedettini et al. 2012; Karska et al. 2013) and high-mass (W11) YSOs. Even when its chemical origin is unclear, the presence of $\mathrm{OH}$ tends to be attributed to shock waves around low- and intermediatemass YSOs (W13; Dionatos et al. 2013; Green et al. 2013). Observing $\mathrm{OH}$ is essential to quantifying the effects of the potentially intense radiation emanating from protostars and of the shock waves propagating in the surrounding regions.

All previous studies of $\mathrm{OH}$, except Wampfler et al. (2010) and W11, were based on observations by the Kuiper Airborne Observatory or the ISO satellite (as listed in W13), or by the PACS receiver onboard the Herschel telescope and were at insufficiently high spectral resolution to enable the $\mathrm{OH}$ emission to be attributed unambiguously to shock waves. This paper presents the first velocity-resolved detection of three triplets of $\mathrm{OH}$ in the high-mass star-forming region Cep A with the GREAT spectrometer onboard SOFIA. We compare these observations with a grid of models, computed with the MHD shock code of Flower \& Pineau des Forêts (2015).

\section{Source selection}

Cepheus A (hereafter Cep A) is a well-known star-forming region, first observed by Sargent (1977), Gyulbudaghian et al. (1978) and Rodriguez et al. (1980). It is located in the Cepheus cloud, at a distance of about 700 pc (Moscadelli et al. 2009). Cep A exhibits numerous manifestations of star formation activity, including peaks of $\mathrm{CO}$ emission, dense molecular clumps, $\mathrm{H}_{2} \mathrm{O}$ and $\mathrm{OH}$ masers, hyper-compact $\mathrm{H}$ II regions, variable radio continuum sources, Herbig-Haro objects, $\mathrm{H}_{2}$ emission, clusters of far-infrared sources, and Class I and II YSOs (see the extensive overview of the region provided in Cunningham et al. 2009). The most spectacular feature is the gigantic anisotropic outflow structure, which is best seen in $\mathrm{H}_{2}$ (e.g. Hodapp 1994), embracing several Herbig-Haro objects (Cunningham et al. 2009) and probably powered by the radio source HW2 (Hughes \& Wouterloot 1982, 1984; Rodriguez et al. 1994), whose luminosity of $10^{4} L_{\odot}$ (Garay et al. 1996) implies a mass of 15-20 $M_{\odot}$. Additionnally, Cunningham et al. (2009) have suggested that the submillimetre and radio continuum source $\mathrm{HW} 3 \mathrm{c}$ might be driving the shocks of the HH168 (the brightest section of the outflow pointing towards the north-west) outflow component, and its counterflow. X-ray emission from the whole region has been mapped by the Chandra/ACIS instrument, revealing three prominent sources in the Cep A east core and a number of other $\mathrm{X}$-ray sources, located in or outside of this core, as appears to be typical of star-forming regions (Pravdo et al. 2009). Figure 1 shows the complexity of the region, as seen by Spitzer/IRAC and in $\mathrm{H}_{2}$ at $2.12 \mu \mathrm{m}$.

\section{Observations}

The observations of the Cep A star-forming region were conducted with the GREAT ${ }^{1}$ spectrometer (Heyminck et al. 2012) during the SOFIA flight on 17 April 2013, as part of Cycle 1 of the programme. One position was observed with coordinates $\alpha_{[\mathrm{J} 2000]}=22^{\mathrm{h}} 56^{\mathrm{m}} 17^{\mathrm{s}} .9, \delta_{[\mathrm{J} 2000]}=+62^{\circ} 01^{\prime} 49^{\prime} .^{\prime} 0$, corresponding to the HW2 source. The receiver was tuned to the frequencies $(1837.817 \mathrm{GHz}$ and $2514.316 \mathrm{GHz})$ of the $\mathrm{OH}$ lines and of the CO (16-15) line (1841.346 GHz). The observed lines were the $\mathrm{OH}$ triplets around 1834.7, 1837.8 , and $2514.3 \mathrm{GHz}$ (see Tables 1 and 2 for spectroscopic details; hereafter triplets at 1835,1838 , and $2514 \mathrm{GHz}$ ), and the CO (16-15) line (see Table 3). Because of spin-orbit interaction, the $\mathrm{OH}$ rotational levels are built within two ladders, ${ }^{2} \Pi_{1 / 2}$ and ${ }^{2} \Pi_{3 / 2}$. Each level is further split by $\Lambda$-doubling and hyperfine structure. The 1835 and $1838 \mathrm{GHz}(163.4$ and $163.1 \mu \mathrm{m})$ transitions are within the ${ }^{2} \Pi_{1 / 2}$ ladder, whereas the ground state one at $2514 \mathrm{GHz}$ $\left(119.4 \mu \mathrm{m}\right.$ ) is within the ${ }^{2} \Pi_{3 / 2}$ ladder (see figure and references in Wampfler et al. 2010). The receiver was connected to a digital XFFTS spectrometer (Klein et al. 2012), providing a bandwidth of $2.5 \mathrm{GHz}$ and resulting in the respective spectral resolutions for these lines: $1.25,1.25,0.91$, and $1.24 \mathrm{~km} \mathrm{~s}^{-1}$ (given in Tables 2 and 3). At these spectral resolutions, the rms uncertainties in the 1835,1838 and $2514 \mathrm{GHz} \mathrm{OH}$ lines and for the $\mathrm{CO}(16-15)$ line were: $0.17,0.12,0.33$, and $0.19 \mathrm{~K}$, respectively. The observations were performed in double beam-switching mode with an amplitude of $80^{\prime \prime}$ (or a throw of $160^{\prime \prime}$ ) at the position angle of $135^{\circ}$ and a phase time of $0.5 \mathrm{~s}$. The nominal focus position was updated regularly against temperature drifts of the

1 GREAT is a development by the MPI für Radioastronomie and the KOSMA/Universität zu Köln, in cooperation with the MPI für Sonnensystemforschung and the DLR Institut für Planetenforschung. 


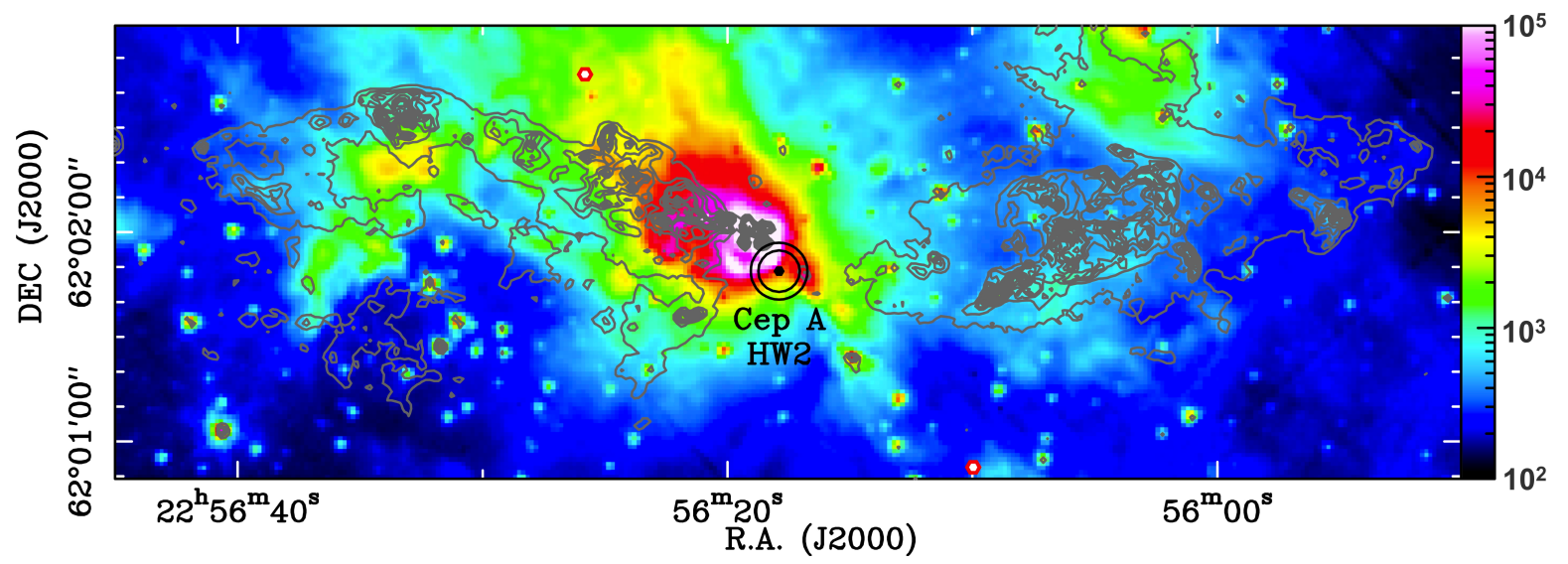

Fig. 1. Cepheus A region as seen in channel 1 of Spitzer/IRAC at $3.6 \mu \mathrm{m}$ (background colours; retrieved from the Spitzer archive; wedge units are $\mathrm{MJy} / \mathrm{sr}$ ), and in $\mathrm{H}_{2}$ at $2.12 \mu \mathrm{m}$ (in dark grey contours, from Cunningham et al. 2009). The observed position "Cep A HW2" is marked by a black hexagon, surrounded by the GREAT beam sizes in CO (16-15) (roughly the same as in $\mathrm{OH} 1835 \mathrm{GHz}$ and $1838 \mathrm{GHz}$ ) and $\mathrm{OH} 2514 \mathrm{GHz}$ (respectively 16". 3 and 11'.9). Multiple OH maser spots have been found in the vicinity of this source by Cohen et al. (1984) and Bartkiewicz et al. (2005). The off positions are also indicated by white and red hexagons in the north-eastern and south-western directions with respect to the observed position.

Table 1. Characteristics of the $\mathrm{OH}$ transitions between the ${ }^{2} \Pi_{1 / 2} J=3 / 2$ and $J=1 / 2$ states, observed in emission, and of the $\mathrm{OH}$ transition between the ${ }^{2} \Pi_{3 / 2} J=5 / 2$ and $J=3 / 2$ states, observed in absorption.

\begin{tabular}{lccccccc}
\hline \hline $\begin{array}{l}\text { Triplet } \\
\text { properties }\end{array}$ & $\begin{array}{c}\text { Transition } \\
F_{p^{\prime}}^{\prime} \rightarrow F_{p}\end{array}$ & $\begin{array}{c}\text { Frequency } \\
(\mathrm{GHz})\end{array}$ & $\begin{array}{c}A_{\mathrm{ul}} \\
\left(\mathrm{s}^{-1}\right)\end{array}$ & $g_{\mathrm{u}}$ & $g_{\mathrm{l}}$ & $\begin{array}{c}E_{\mathrm{u}} \\
(\mathrm{K})\end{array}$ & $\begin{array}{c}\text { Shift } \\
\left(\mathrm{km} \mathrm{s}^{-1}\right)\end{array}$ \\
\hline Signal & $1+\rightarrow 1-$ & 1837.7466 & $2.1(-2)$ & 3 & 3 & 270.1 & 11.5 \\
$1838 \mathrm{GHz}$ & $2+\rightarrow 1-$ & 1837.8168 & $6.4(-2)$ & 5 & 3 & 270.1 & 0.0 \\
$163.1 \mu \mathrm{m}$ & $1+\rightarrow 0-$ & 1837.8370 & $4.3(-2)$ & 3 & 1 & 270.1 & -3.3 \\
\hline Image & $1-\rightarrow 1+$ & 1834.7355 & $2.1(-2)$ & 3 & 3 & 269.8 & 1.9 \\
$1835 \mathrm{GHz}$ & $2-\rightarrow 1+$ & 1834.7474 & $6.4(-2)$ & 5 & 3 & 269.8 & 0.0 \\
$163.4 \mu \mathrm{m}$ & $1-\rightarrow 0+$ & 1834.7504 & $4.2(-2)$ & 3 & 1 & 269.8 & -0.5 \\
\hline Signal & $2-\rightarrow 2+$ & 2514.29873 & $1.4(-2)$ & 5 & 5 & 120.7 & 2.1 \\
$2514 \mathrm{GHz}$ & $3-\rightarrow 2+$ & 2514.31670 & $1.4(-1)$ & 7 & 5 & 120.7 & 0.0 \\
$119.2 \mu \mathrm{m}$ & $2-\rightarrow 1+$ & 2514.35349 & $1.2(-1)$ & 5 & 3 & 120.7 & -4.5 \\
\hline
\end{tabular}

Notes. $A(B) \equiv A \times 10^{B}$. The "shift" column contains the velocity shift relative to the component with the largest Einstein $A$ coefficient. Source: JPL (Pickett et al. 1998).

Table 2. Observational parameters of the $\mathrm{OH}$ transitions between the ${ }^{2} \Pi_{1 / 2} J=3 / 2$ and $J=1 / 2$ states, observed in emission around 1835 and $1838 \mathrm{GHz}$, and of the $\mathrm{OH}$ transition between the ${ }^{2} \Pi_{3 / 2} J=5 / 2$ and $J=3 / 2$ states, observed in absorption around $2514 \mathrm{GHz}$.

\begin{tabular}{|c|c|c|c|c|c|c|c|c|c|}
\hline $\begin{array}{l}\text { Triplet } \\
\text { properties }\end{array}$ & $\begin{array}{l}\text { Transition } \\
F_{p^{\prime}}^{\prime} \rightarrow F_{p}\end{array}$ & $\begin{array}{l}\text { Frequency } \\
\quad(\mathrm{GHz})\end{array}$ & $\begin{array}{l}\text { Beam size } \\
\left({ }^{\prime \prime}\right)\end{array}$ & $\begin{array}{c}\text { Observing } \\
\text { date }\end{array}$ & $\begin{array}{l}\text { Integration time } \\
\text { (on source; } s \text { ) }\end{array}$ & $\begin{array}{l}\text { Spectral resolution } \\
\left(\mathrm{km} \mathrm{s}^{-1}\right)\end{array}$ & $\begin{array}{l}\text { Beam } \\
\text { efficiency }\end{array}$ & $\begin{array}{l}\text { Forward } \\
\text { efficiency }\end{array}$ & $\begin{array}{l}T_{\text {sys }} \\
(\mathrm{K})\end{array}$ \\
\hline Signal & $1+\rightarrow 1-$ & 1837.7466 & & & & & & & \\
\hline $\begin{array}{l}1838 \mathrm{GHz} \\
163.1 \mu \mathrm{m}\end{array}$ & $\begin{array}{l}2+\rightarrow 1- \\
1+\rightarrow 0-\end{array}$ & $\begin{array}{l}1837.8168 \\
1837.8370\end{array}$ & 16.3 & $17 / 04 / 13$ & 228 & 1.25 & 0.67 & 0.97 & 2689 \\
\hline Image & $1-\rightarrow 1+$ & 1834.7355 & & & & & & & \\
\hline $\begin{array}{l}1835 \mathrm{GHz} \\
163.4 \mu \mathrm{m}\end{array}$ & $\begin{array}{l}2-\rightarrow 1+ \\
1-\rightarrow 0+\end{array}$ & $\begin{array}{l}1834.7474 \\
1834.7504\end{array}$ & 16.3 & $17 / 04 / 13$ & 228 & 1.25 & 0.67 & 0.97 & 2689 \\
\hline Signal & $2-\rightarrow 2+$ & 2514.298730 & & & & & & & \\
\hline $2514 \mathrm{GHz}$ & $3-\rightarrow 2+$ & 2514.316705 & 11.9 & $17 / 04 / 13$ & 378 & 0.91 & 0.70 & 0.97 & 9687 \\
\hline
\end{tabular}

telescope structure. The pointing was established with the optical guide cameras to an accuracy of $\sim 5^{\prime \prime}$. The beam widths and efficiencies are indicated in Tables 2 (for the $\mathrm{OH}$ transitions) and 3 (for the $\mathrm{CO}$ transition). The data were calibrated with the KOSMA/GREAT calibrator (Guan et al. 2012), removing residual telluric lines, and further processed with the CLASS software $^{2}$. This processing mostly consisted of linear baseline removal. For the $\mathrm{OH} 2514 \mathrm{GHz}$ line, the continuum temperature

\footnotetext{
2 http://www.iram.fr/IRAMFR/GILDAS
}

was approximately $\sim 9 \mathrm{~K}$, whereas all the other lines had a baseline at the $\sim 6 \mathrm{~K}$ level.

\section{Results}

\section{1. $\mathrm{OH}$ in emission}

The emission in the two triplets at 1835 and $1838 \mathrm{GHz}$ from the Cep A HW2 position can be seen in the two upper panels of Fig. 2. The frequencies of the components of each triplet 
Table 3. CO (16-15) line and observational parameters.

\begin{tabular}{cc}
\hline \hline$A_{\mathrm{ul}}\left(\mathrm{s}^{-1}\right)$ & $4.05 \times 10^{-4}$ \\
$\nu(\mathrm{GHz})$ & 1841.345506 \\
$E_{\mathrm{u}}(\mathrm{K})$ & 751.72 \\
$\lambda(\mu \mathrm{m})$ & 162.81 \\
\hline Beam size (") & 16.3 \\
Observing date & $17 / 04 / 13$ \\
Integration time (on source; s) & 180 \\
Spectral resolution $\left(\mathrm{km} \mathrm{s}^{-1}\right)$ & 1.24 \\
$B_{\text {eff }}$ & 0.67 \\
$F_{\text {eff }}$ & 0.97 \\
$T_{\text {sys }}(\mathrm{K})$ & 2742 \\
\hline
\end{tabular}

Notes. Spectroscopic data source: CDMS (Müller et al. 2001, 2005).

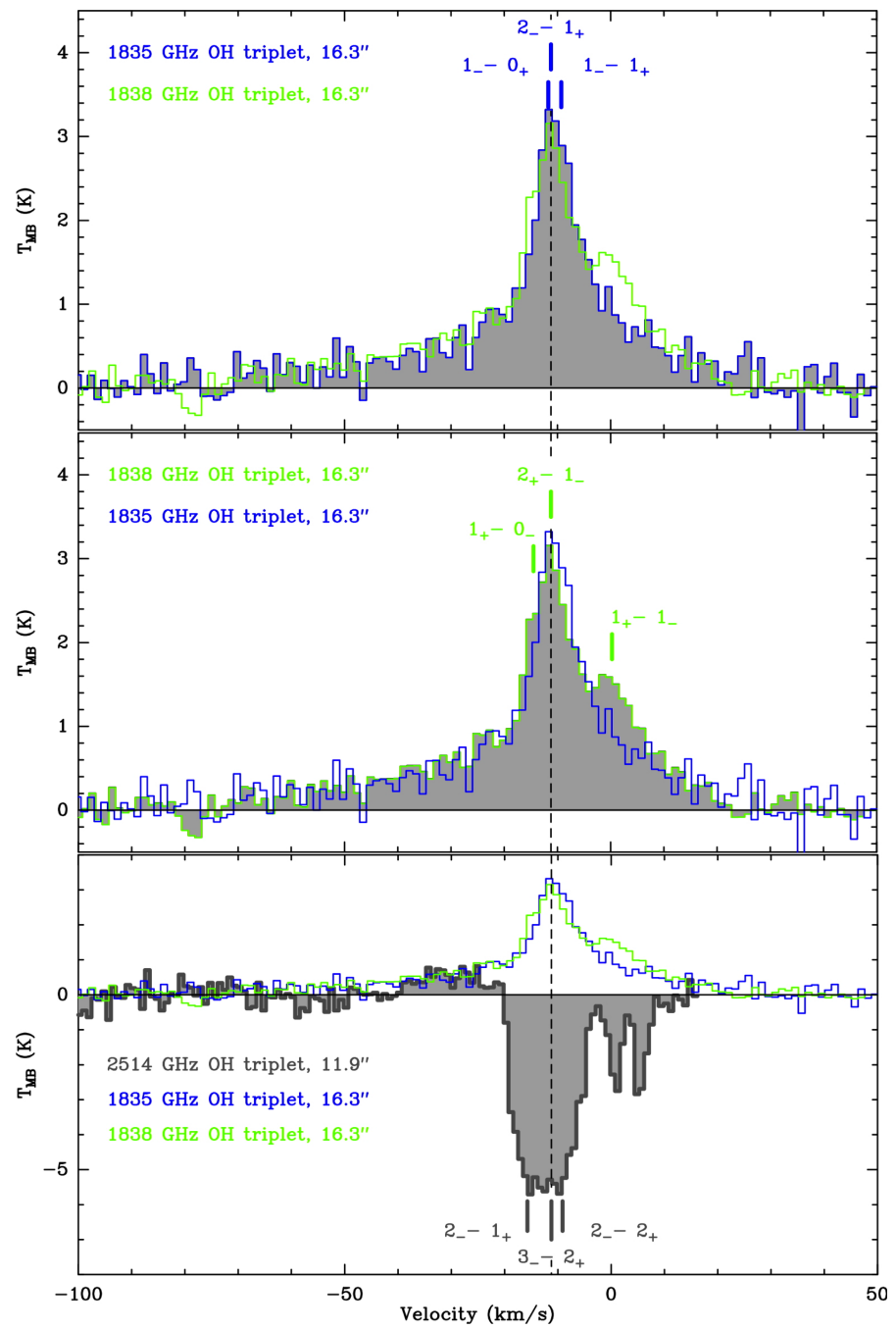

Fig. 2. Top panel: $1835 \mathrm{GHz} \mathrm{OH}$ triplet (blue line and grey histogram), overlaid with the $1838 \mathrm{GHz}$ triplet (green line). Middle panel: the $1838 \mathrm{GHz} \mathrm{OH}$ triplet (green line and grey histogram), overlaid with the $1835 \mathrm{GHz}$ triplet (blue line). Bottom panel: the $2514 \mathrm{GHz} \mathrm{OH}$ triplet (dark grey and grey histograms), overlaid with the $1838 \mathrm{GHz}$ (green line) and $1835 \mathrm{GHz}$ (blue line) triplets. In all panels, the lines were observed at the Cep A HW2 position, indicated in Fig. 1, and the three components of the triplets are indicated in the same colour as the considered triplet: see Tables 1 and 2. The vertical dashed line is at the $v_{\mathrm{lsr}}$ of the cloud $\left(-11.2 \mathrm{~km} \mathrm{~s}^{-1}\right.$; e.g. Narayanan \& Walker 1996; Gómez et al. 1999). In the top and middle panels, the spatial and spectral resolutions are $16 . ' 3$ and $1.25 \mathrm{~km} \mathrm{~s}^{-1}$, respectively. In the bottom panel, they are $11^{\prime \prime} .9$ and $0.91 \mathrm{~km} \mathrm{~s}^{-1}$. are indicated; the $1_{+}-1_{-}$component of the $1838 \mathrm{GHz}$ triplet is partially resolved. Both the triplets peak at the $v_{\text {lsr }}$ of the cloud (-11.2 km s${ }^{-1}$; e.g., Narayanan \& Walker 1996; Gómez et al. 1999), and exhibit high-velocity wings in the blueshifted and redshifted directions (respectively extending up to $\sim-75$ and to $\sim 35 \mathrm{~km} \mathrm{~s}^{-1}$ ), which are indicative of the presence of highvelocity shocks in the region. Since the $1838 \mathrm{GHz}$ line profile is slightly more complex (the $1+\rightarrow 1-$ component has a relatively large velocity shift of $11.5 \mathrm{~km} \mathrm{~s}^{-1}$, see Table 1), we applied a Gaussian fit to the $1835 \mathrm{GHz}$ line alone, with no accounting for its hyperfine structure. We found that this line can be fitted by a combination of two Gaussian components: one narrow $\left(\Delta v \approx 8.4 \mathrm{~km} \mathrm{~s}^{-1}\right)$, peaking at $-10.3 \mathrm{~km} \mathrm{~s}^{-1}$; the other broad $\left(\Delta v \approx 50.4 \mathrm{~km} \mathrm{~s}^{-1}\right)$, peaking at $-11.8 \mathrm{~km} \mathrm{~s}^{-1}$. Such a doubleGaussian structure has been obtained by W13 in another massive SFR, W3-IRS5, whereas lower mass SFRs seem to exhibit only a broad component (e.g. Wampfler et al. 2010 for this conclusion, and Kristensen et al. 2013 for the example of Ser SMM1). The quality of the fits of the $\mathrm{OH}$ lines is not decreased by requiring the peak velocities of all the Gaussian components to be $-11.2 \mathrm{~km} \mathrm{~s}^{-1}$, the velocity of the source. The parameters of both of the double-Gaussian fits are given in Table 4.

\section{2. $\mathrm{OH}$ in absorption}

The profile of the triplet at $2514 \mathrm{GHz}$ (see Table 1) from the Cep A HW2 position is shown in the bottom panel of Fig. 2. A baseline has been removed, since the continuum temperature at this frequency was $\sim 9 \mathrm{~K}$ (see Sect. 3). The triplet structure is not resolved, but three velocity components can be seen in absorption. One peaks at the cloud velocity, showing the presence of $\mathrm{OH}$ in the ambient cloud; two other line-of-sight clouds must give rise to the absorption features at $1.2 \mathrm{~km} \mathrm{~s}^{-1}$ and $4.8 \mathrm{~km} \mathrm{~s}^{-1}$, although we have found no record in the literature of observations of the corresponding absorption components in other species. Neither of these velocity components is present in the other $\mathrm{OH}$ triplets. No evidence could be found of another species or line likely to cause absorption at these frequencies. Finally, the emission seen in the blue part of the $2514 \mathrm{GHz}$ profile is at $2.5 \sigma$ level in several velocity channels between -35 and $-25 \mathrm{~km} \mathrm{~s}^{-1}$. It coincides with the wings of the triplets seen in emission, despite the difference in angular resolution. It is therefore likely to be associated with the blueshifted shocked gas.

\section{3. $C O(16-15)$ line emission}

We also obtained one velocity-resolved CO (16-15) spectrum in Cep A HW2 that we present in Fig. 3. The parameters of the line and of the telescope at this frequency are given in Table 3. The line has a similar structure to the $\mathrm{OH}$ triplets: it peaks at the $v_{\text {lsr }}$ of the cloud and has line wings typical of shocks in the blueshifted and redshifted directions (respectively extending up to $\sim-75$ and to $\sim 35 \mathrm{~km} \mathrm{~s}^{-1}$ ). Very similar (double- or triple-) Gaussian decompositions can be applied to the $\mathrm{OH}$ line at $1835 \mathrm{GHz}$ (see Table 4). This is after the narrow emission feature at $\sim 10.7 \mathrm{~km} \mathrm{~s}^{-1}$ is separately fitted by a single Gaussian component (with parameters $\Delta v \sim 1 \mathrm{~km} \mathrm{~s}^{-1}, v_{\text {peak }}=10.7 \mathrm{~km} \mathrm{~s}^{-1}$, and $T_{\text {peak }} \sim 1.7 \mathrm{~K}$ ). This feature is due to mesospheric $\mathrm{CO}$ over-compensated for by the correction for the atmospheric opacity. We found the CO line can be fitted by two Gaussians of width $7.1 \mathrm{~km} \mathrm{~s}^{-1}$ and $36.2 \mathrm{~km} \mathrm{~s}^{-1}$, centred at -10.9 and $-12.7 \mathrm{~km} \mathrm{~s}^{-1}$, respectively (see Table 4). The CO line also peaks at the cloud velocity (contrary to the corresponding spectrum of 
Table 4. Parameters of the Gaussian decompositions of the $\mathrm{OH} 1835 \mathrm{GHz}$ and $\mathrm{CO}(16-15)$ lines.

\begin{tabular}{|c|c|c|c|c|c|c|c|c|c|c|}
\hline & \multicolumn{4}{|c|}{ Double-Gaussian decomposition } & \multicolumn{6}{|c|}{ Triple-Gaussian decomposition } \\
\hline & \multicolumn{2}{|c|}{ OH 1835 GHz } & \multicolumn{2}{|c|}{$\mathrm{CO}(16-15)$} & \multicolumn{3}{|c|}{ OH $1835 \mathrm{GHz}$} & \multicolumn{3}{|c|}{$\mathrm{CO}(16-15)$} \\
\hline & Narrow & Broad & Narrow & Broad & Blue & Ambient & Red & Blue & Ambient & Red \\
\hline$\Delta v\left(\mathrm{~km} \mathrm{~s}^{-1}\right)$ & 8.4 & 50.4 & 7.1 & 36.2 & 53.6 & 7.8 & 15.2 & 48.1 & 7.3 & 5.3 \\
\hline$v_{\text {peak }}\left(\mathrm{km} \mathrm{s}^{-1}\right)$ & -10.3 & -11.8 & -10.9 & -12.7 & -14.7 & -10.6 & -2.6 & -17.2 & -11.0 & -3.0 \\
\hline$T_{\text {peak }}(\mathrm{K})$ & 2.3 & 0.9 & 4.9 & 1.2 & 0.7 & 2.4 & 0.3 & 0.8 & 5.3 & 0.7 \\
\hline rms base $(\mathrm{K})$ & \multicolumn{2}{|c|}{0.17} & \multicolumn{2}{|c|}{0.19} & \multicolumn{3}{|c|}{0.16} & \multicolumn{3}{|c|}{0.19} \\
\hline rms signal (K) & \multicolumn{2}{|c|}{0.19} & \multicolumn{2}{|c|}{0.21} & \multicolumn{3}{|c|}{0.18} & \multicolumn{3}{|c|}{0.21} \\
\hline
\end{tabular}

Notes. "rms base" and "rms line" denote the rms values associated with the residual. When fitting the lines with a double Gaussian, forcing the $v_{\text {peak }}$ of one or both of the components to the $v_{\mathrm{lsr}}$ of the cloud leads to no more than $\sim 12 \%$ variations in the rms values.

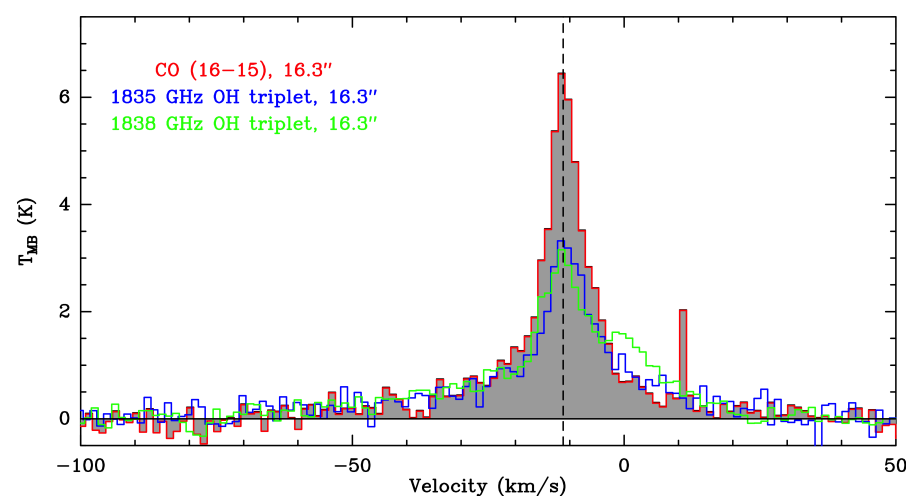

Fig. 3. CO (16-15) transition (red line and grey histograms) at the Cep A HW2 position, overlaid with the $1835 \mathrm{GHz}$ and $1838 \mathrm{GHz}$ triplets (blue and green lines, from Fig. 2). The spatial and spectral resolutions of the CO line are 16.3 and $1.24 \mathrm{~km} \mathrm{~s}^{-1}$. The vertical dashed line is at the cloud $v_{\mathrm{lsr}}$ of $-11.2 \mathrm{~km} \mathrm{~s}^{-1}$ (Narayanan \& Walker 1996; Gómez et al. 1999).

Ser SMM1: Kristensen et al. 2013). As for the $\mathrm{OH} 1835 \mathrm{GHz}$ line, the quality of the fits of the CO line is not decreased by requiring the peak velocities of all the Gaussian components to be $-11.2 \mathrm{~km} \mathrm{~s}^{-1}$, the velocity of the source. The similarity of the line wings in both of the $\mathrm{OH}$ emitting triplets and the $\mathrm{CO}$ lines suggests that the observed $\mathrm{OH}$ emission comes from the same gas as the CO (16-15) line and indicates the presence of highvelocity shocks in the observed region. The intensities integrated over the blue, red, and total velocity ranges (see Table 5 for the corresponding values) are $43.5 \mathrm{~K} \mathrm{~km} \mathrm{~s}^{-1}, 41.9 \mathrm{~K} \mathrm{~km} \mathrm{~s}^{-1}$, and $85.3 \mathrm{~K} \mathrm{~km} \mathrm{~s}^{-1}$.

\section{Discussion}

In this section, we present an analysis of our observations, based on a comparison of the integrated intensities of $\mathrm{OH}$ and $\mathrm{CO}$ lines with the values computed by the shock model of Flower \& Pineau des Forêts $(2013,2015)$. The underlying assumption is that the $\mathrm{OH}$ and $\mathrm{CO}$ emission stems from shocked gas. We note that no $\mathrm{H}_{2}$ emission is detected at $2.12 \mu \mathrm{m}$ in our observed region. This does not contradict our assumption, but rather reflects the high extinction associated with the HW sources in the region $\left(A_{\mathrm{v}}=500\right.$ to 1000 , Cunningham et al. 2009). For instance, a microjet was recently imaged at high spatial resolution in the HH212 protostellar outflow by Codella et al. (2014) in $\mathrm{SiO}$ and Podio et al. (2015) in $\mathrm{SO}$ and $\mathrm{SO}_{2}$, without having a counterpart in $\mathrm{H}_{2}$ emission at $2.12 \mu \mathrm{m}$. Our modelling method is the same as presented in Gusdorf et al. (2011, 2012). The model is one-dimensional, can simulate the propagation of stationary $\mathrm{C}$ - and J-type shocks, and includes a self-consistent
Large Velocity Gradient (LVG) treatment of the radiative transfer in the lines emitted by various cooling species $\left(\mathrm{CO}, \mathrm{H}_{2} \mathrm{O}\right.$, $\mathrm{SiO}, \mathrm{NH}_{3}$, and $\mathrm{OH}$ ). The isotropic approximation was used for the escape probability with $\beta=\left(1-\mathrm{e}^{-\tau_{\perp}}\right) / \tau_{\perp}, \tau_{\perp}$ being the LVG opacity in the direction perpendicular to the shock front (e.g. Gusdorf et al. 2008). Solving the radiative transfer in the lines within the shock model is more precise than an LVG postprocessing of the shock model's output files. Indeed, it allows the level populations to be computed under the steady state assumption $(\mathrm{d} n / \mathrm{d} t=v \cdot \partial n / \partial z)$ instead of under the statistical equilibrium one $(\mathrm{d} n / \mathrm{d} t=0)$. The range of input parameters covered by our calculations was as follows: pre-shock densities $n_{\mathrm{H}}=n(\mathrm{H})+2 n\left(\mathrm{H}_{2}\right)=10^{4}, 10^{5}, 10^{6} \mathrm{~cm}^{-3}$; transverse magnetic field strengths $B(\mu \mathrm{G})=b\left[n_{\mathrm{H}}\left(\mathrm{cm}^{-3}\right)\right]^{1 / 2}$, where the parameter $b=1$ for C-type and $b=0.1$ for J-type shocks; and shock velocities $v_{\mathrm{s}}=10,15,20,25,30,35 \mathrm{~km} \mathrm{~s}^{-1}$. In the high-density regions of the Cep A outflow, the relation between the total magnetic field strength and the density was observationally shown to be $B(\mu \mathrm{G}) \propto\left[n_{\mathrm{H}}\left(\mathrm{cm}^{-3}\right)\right]^{0.47}$ by Vlemmings (2008), based on a collection of $\mathrm{OH}, \mathrm{NH}_{3}, \mathrm{CH}_{3} \mathrm{OH}$, and $\mathrm{H}_{2} \mathrm{O}$ maser measurements. We adopted the same law with a proportionality factor of 1 for the transverse magnetic field strength. Grain-grain interactions, such as studied by e.g. Guillet et al. (2011), are not included in our model. The maximum shock velocities in the grid are determined by the following considerations: C-type shock waves cannot propagate above a critical shock speed, which is, for example, $32 \mathrm{~km} \mathrm{~s}^{-1}$ for $b=1$ and $n_{\mathrm{H}}=10^{6} \mathrm{~cm}^{-3}$ (Flower \& Pineau des Forêts 2003). Additionally, when $\mathrm{H}_{2}-$ the main coolant - becomes dissociated, a thermal runaway occurs that prevents the model from converging towards a cold, compressed post-shock medium. In this case, no C-type shock can propagate, only a J-type one (Le Bourlot et al. 2002).

\subsection{Scenario 1: one outflow, two shock layers}

As in the case of other molecular species (e.g. SiO: Gusdorf et al. 2008; Anderl et al. 2013), the double-Gaussian decomposition of the $\mathrm{OH}$ line profiles (Sect. 4.1) fails to account for the complexity of the observed emission. Most likely, the observed line profiles are produced by a collection of shocked layers. The statistical modelling of such a collection has been shown by Lesaffre et al. (2013) to involve a number of free parameters. Given the limited number of observational constraints, we first adopted the simplifying assumption that the line profiles are the result of two shocks, propagating in the blueshifted and redshifted directions (as in Gusdorf et al. 2012), together with an "ambient" component, and fitted the $\mathrm{OH}$ and $\mathrm{CO}$ line profiles by a combination of three Gaussians; the corresponding parameters are given in Table 4, and the corresponding figures are shown in the Appendix (Figs. A.1 and A.2). This approach resulted in slightly 
A\&A 585, A45 (2016)

Table 5. Observed integrated intensities $\left(\int T_{\mathrm{MB}} \mathrm{d} v\right.$, in $\left.\mathrm{K} \mathrm{km} \mathrm{s}^{-1}\right)$ of the emission lines.

\begin{tabular}{lcccccc}
\hline \hline & \multicolumn{3}{c}{ Blueshifted } & \multicolumn{3}{c}{ Redshifted } \\
Component & HV & LV & total & LV & HV & total \\
Velocity interval $\left(\mathrm{km} \mathrm{s}^{-1}\right)$ & {$[-90 ;-19]$} & {$[-19 ;-11]$} & {$[-90 ;-11]$} & {$[-11 ;-3]$} & {$[-3 ; 30]$} & {$[-11 ; 30]$} \\
Corresponding $\Delta v\left(\mathrm{~km} \mathrm{~s}^{-1}\right)$ & 71 & 8 & 79 & 8 & 33 & 41 \\
\hline OH 1835 GHz & 20.7 & 15.5 & 36.2 & 18.1 & 15.5 & 33.6 \\
OH 1838 GHz & 20.4 & 16.7 & 37.1 & 16.3 & 18.8 & 35.1 \\
OH 2514 GHz & $21.5^{a}$ & - & - & - & - & - \\
\hline CO $(16-15)$ & 16.4 & 27.1 & 43.5 & 28.0 & 13.9 & 41.9 \\
\hline
\end{tabular}

Notes. The uncertainty in the integrated intensities mostly comes from the line temperature calibration of the order of $\pm 10 \%$. LV and HV stand for low-velocity and high-velocity. The "total" value was used in scenario 1 (Sect. 5.1), while the LV and HV values were used in scenario 2 (Sect. 5.2). ${ }^{(a)}$ Integrated between -90 and $-20 \mathrm{~km} \mathrm{~s}^{-1}$.

better residual rms than the double-Gaussian fits. This approach was used only to infer an estimate of the shock velocity: when a line is fitted by the combination of narrow and broad Gaussian components, it does not mean that the shock is only responsible for the broad emission. $\mathrm{OH}$ emission from the shock is expected at velocities close to the velocity of the source and contributes to the narrow component.

The triple-Gaussian decomposition indicates that the $\mathrm{OH}$ spectral lines are slightly wider than the $\mathrm{CO}$ line. This could be due to the hyperfine structure of the $\mathrm{OH}$ lines or to the uncertainty on the fit and on the baseline correction. It also indicates a very high FWHM for the blueshifted Gaussian component: $53.6 \mathrm{~km} \mathrm{~s}^{-1}$ for $\mathrm{OH}$ and $48.1 \mathrm{~km} \mathrm{~s}^{-1}$ for $\mathrm{CO}$, corresponding to a calculated full width at tenth maximum (FWTM) of $97.7 \mathrm{~km} \mathrm{~s}^{-1}$ and $87.7 \mathrm{~km} \mathrm{~s}^{-1}$, respectively. If generated by a single shock - which is unlikely - this magnitude of velocity is typical of shocks with radiative precursors, which lie outside the scope of our models. If generated by a collection of shocks, the number of observational constraints is too small to permit a meaningful analysis. We therefore decided to exclude this velocity component from our analysis in this first approach. On the other hand, the velocities observed in the redshifted component fall within the domain of applicability of the shock code: FWHM and FWTM of $15.2 \mathrm{~km} \mathrm{~s}^{-1}$ and $27.7 \mathrm{~km} \mathrm{~s}^{-1}$, respectively, for $\mathrm{OH}$ and $5.3 \mathrm{~km} \mathrm{~s}^{-1}$ and $9.7 \mathrm{~km} \mathrm{~s}^{-1}$ for $\mathrm{CO}$. In the case of $\mathrm{OH}$, the full width at one percent of maximum is $39.2 \mathrm{~km} \mathrm{~s}^{-1}$. Accordingly, we attributed a velocity between $27.7 \mathrm{~km} \mathrm{~s}^{-1}$ and $41 \mathrm{~km} \mathrm{~s}^{-1}$ to the red-wing shock; the latter value is slightly greater than $39.2 \mathrm{~km} \mathrm{~s}^{-1}$, reflecting the observed red-wing emission (from approximately -11 to $30 \mathrm{~km} \mathrm{~s}^{-1}$, regardless of the type of Gaussian fit). The intensity was integrated over this velocity range and is given in Table 5.

The comparison of the observations with the models is made in Fig. 4. In each panel, the abscissa is the shock velocity of the model. The (large) uncertainty in the observed shock velocity is indicated by the horizontal error bar. In the context of onedimensional modelling, adopted here, the shock is assumed to be seen face-on. However, it is possible that shocks propagate in an already moving medium or that the flow is orientated towards the plane of the sky, resulting in, respectively, lower or higher shock velocities. In the former case, the observed integrated intensity should be compared to models with a lower velocity than the values inferred from our Gaussian fits, i.e. less than $27.7 \mathrm{~km} \mathrm{~s}^{-1}$. In the latter case, the velocity should exceed $41 \mathrm{~km} \mathrm{~s}^{-1}$ and is likely to lie outside the domain of applicability of the model, and hence of the scope of this study. Furthermore, both effects could intervene (an inclined shock propagating in a moving medium).

We note that varying the assumed inclination of the shock front has consequences for the line temperatures, calculated along the line of sight. In Appendix B, we provide a brief assess- ment of this effect for one J-type and one C-type model from the grid.

The ordinate in Fig. 4 is the observed (or predicted) integrated intensity. The uncertainty in the integrated intensities is about the size of the filled circles that represent the observations. The upper and lower panels present C- and J-type shock models, respectively. The left-hand side, central, and right-hand side panels respectively present the comparisons for $\mathrm{OH}$ in emission (both at 1835 and $1838 \mathrm{GHz}$ ), $\mathrm{OH}$ in absorption, and CO (16-15). In the central panels, the purple line is the observed intensity, integrated between -90 and $-20 \mathrm{~km} \mathrm{~s}^{-1}$. This velocity range corresponds to the blueshifted shock component and is not to be considered in this first part of our analysis. All the shock models in our grid predict somewhat more emission in the $1838 \mathrm{GHz}$ than in the $1835 \mathrm{GHz}$ line, as is observed.

The main conclusion from Fig. 4 is that, under the assumption that $\mathrm{CO}$ and $\mathrm{OH}$ arise from the same gas component, no shock model from our grid can fit all the observations, even with a filling factor equal to its upper limit of 1 . The trend that one perceives in this figure suggests that only a dense $\left(n_{\mathrm{H}}>10^{5} \mathrm{~cm}^{-3}\right)$ and fast $\left(v_{\mathrm{s}} \gtrsim 30 \mathrm{~km} \mathrm{~s}^{-1}\right)$ J-type shock model could conceivably fit the data with a filling factor close to the unity for both $\mathrm{OH}$ and $\mathrm{CO}$. Unfortunately we were unable to run such a case because of the numerical limitations of the model. These conclusions are not modified by varying the inclination angle, as indicated by our study presented in Appendix B. If confirmed, this would mean that the $\mathrm{OH}$ and high- $J \mathrm{CO}$ emission originates in the strongest shock components within the beam, which are more likely to be in the jet than in the outflow cavity walls. We note that, in the densest and fastest J-type model $\left(n_{\mathrm{H}}=10^{5} \mathrm{~cm}^{-3}\right.$ and $\left.v_{\mathrm{s}}=30 \mathrm{~km} \mathrm{~s}^{-1}\right)$, the optical depth of the $\mathrm{OH}$ line at $2514 \mathrm{GHz}$ locally reaches high values $(\tau \approx 35-40)$.

Under high-density, high-temperature conditions, one would expect grain-grain interactions (fragmentation, in particular) to play a significant part in the thermal structure of the shock (e.g. Guillet et al. 2009, 2011). Anderl et al. (2013) have shown that including them does not significantly alter the predicted $\mathrm{OH}$ emission of C-type models with $n_{\mathrm{H}}$ of the order of $10^{5} \mathrm{~cm}^{-3}$ (see their Fig. B.4), but that it does affect the shape of the predicted energy distribution of the $\mathrm{CO}$ spectral line (see their Fig. B.2). Furthermore, it is likely that, at such high shock velocities, a so-called radiative precursor develops ahead of the shock wave, which heats, dissociates, and ultimately ionizes the pre-shock gas (Hollenbach \& McKee 1989); this phenomenon is not included in the model employed here. From the results in Fig. 4, we see that a dense and fast J-type solution could be combined with a less dense C-type solution (with $n_{\mathrm{H}}$ in the range $\left[10^{4}-10^{5}\right] \mathrm{cm}^{-3}$ and $v_{\mathrm{s}} \gtrsim 30 \mathrm{~km} \mathrm{~s}^{-1}$ ), without significantly altering the comparison with the observations. This could mean that either the emission stems from a young, non-stationary shock or 
A. Gusdorf et al.: $\mathrm{OH}$ emission in the high-mass star-forming region Cepheus A
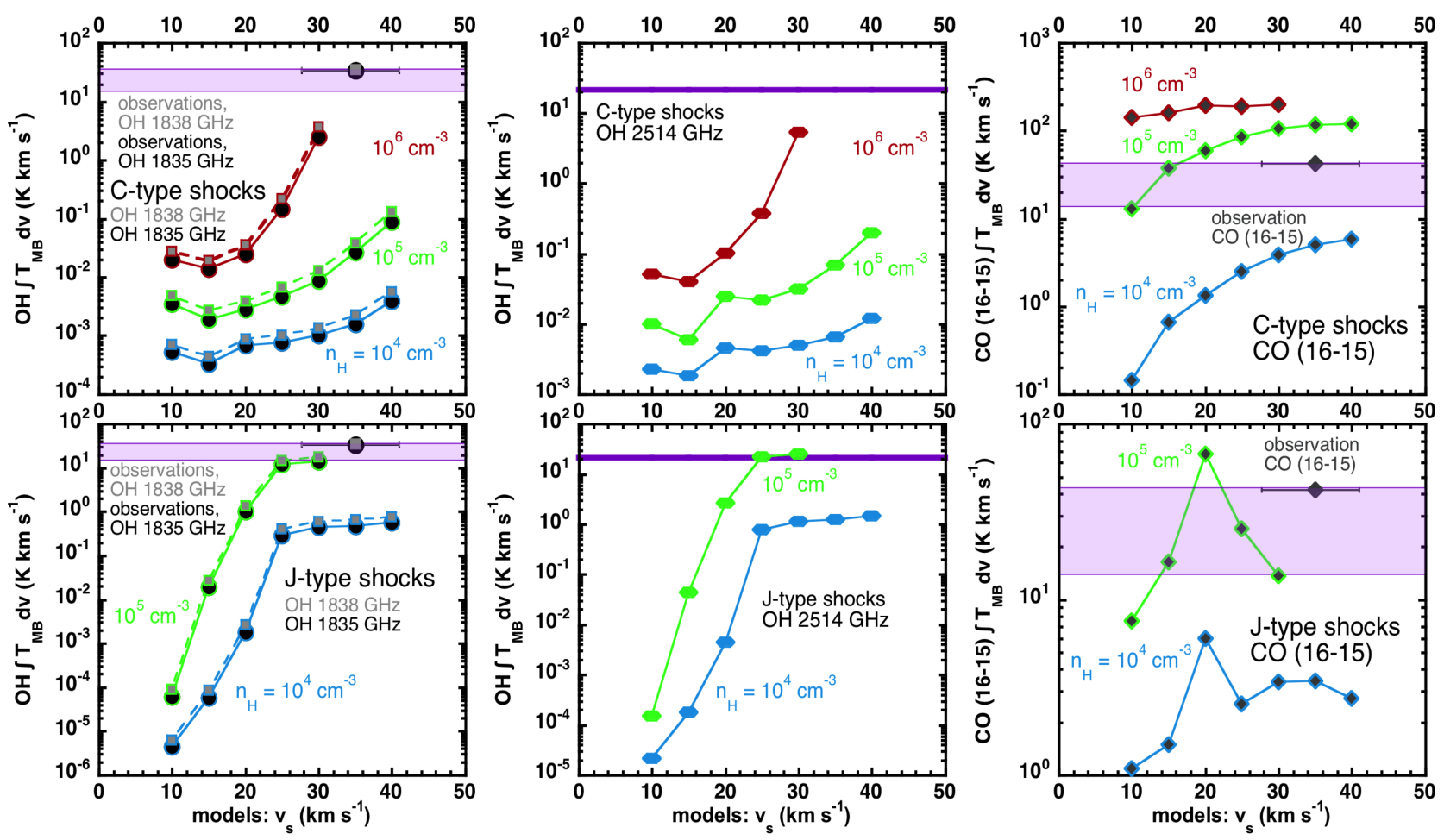

Fig. 4. Comparisons of the integrated intensities (main beam temperature units) of all observed lines with the predictions of our grid of C-type (upper panels) and J-type (lower panels) models. In all panels, the abscissa is the shock velocity. In all panels, the colour symbols are the model predictions, where the red, blue, and green filled circles correspond to the pre-shock densities indicated in each panel. Finally, in all panels, the grey and black points are the observations integrated over the whole redshifted shock component (scenario 1, Sect. 5.1), while the pink area corresponds to the range of values that correspond to the LV and HV values, see Table 5, in turn corresponding to scenario 2, Sect. 5.2. In the central panels, the purple line is the observed intensity integrated between -90 and $-20 \mathrm{~km} \mathrm{~s}^{-1}$. Left-hand side panels: $\mathrm{OH}$ at $\sim 1835 \mathrm{GHz}$ and $\sim 1838 \mathrm{GHz}$. The computed $1835 \mathrm{GHz}$ and $1838 \mathrm{GHz}$ intensities are connected by continuous and broken curves, respectively. The observed intensities are indicated by black $(1835 \mathrm{GHz})$ and grey $(1838 \mathrm{GHz})$ points with horizontal bars, reflecting the uncertainty on the shock velocity; the uncertainty on the intensity are of the order of the points sizes (see text). Central panels: $\mathrm{OH}$ at 2514 GHz. Right-hand side panels: CO (16-15).

alternatively, that it stems from both the jet (J-type contribution) and the bowshock or the outflows cavity walls it has generated (C-type contribution). From this perspective, it is interesting to note that a solution based on a combination of C- and J-type models has been found by Flower et al. (2003) to fit the molecular $\mathrm{H}_{2}$ observations of Wright et al. (1996) in a position (Cep A West) located $15^{\prime \prime}$ away from the presently studied one.

\subsection{Scenario 2: two outflows or one outflow with cavity walls, four shock layers}

Cunningham et al. (2009) discuss the possibility that two outflows could be present in the beam of their CO observations, one being responsible for low-velocity emission (less than $8 \mathrm{~km} \mathrm{~s}^{-1}$ away from the $v_{\text {lsr }}$ ), the other for higher-velocity emission (more than $8 \mathrm{~km} \mathrm{~s}^{-1}$ away from the $v_{\mathrm{lsr}}$ ). They also indicate that this low-velocity emission could originate in the walls of a largescale bipolar cavity. In either case, at least four shocks would be propagating within our observing beam, all with filling factors close to the unity. Lacking more precise information, we attribute the line emission to four velocity ranges - one (socalled LV) of low- and one (so-called HV) of high-velocity for the blueshifted and the redshifted emission. The intensity of the $\mathrm{OH}$ line at $2514 \mathrm{GHz}$, which is integrated between -90 and $-20 \mathrm{~km} \mathrm{~s}^{-1}$ and indicated by a purple line in the central panels, thus roughly corresponds to the blue HV range. It could be a lower limit to the real value, owing to absorption at the velocities in the range closest to the source's velocity. The definitions of the velocity ranges and corresponding integrated intensities are given in Table 5.
Except for the $2514 \mathrm{GHz} \mathrm{OH}$ line, the line intensities, integrated over the different velocity ranges, are similar in magnitude. When presenting these results in Fig. 4, we show only the minimum and the maximum values of the integrated intensities in the four velocity ranges (the pink horizontal lines) and shade the interval thus defined in pink. We chose to extend the pink band over the whole shock-velocity range of the panels because the width of the HV or LV emission is not necessarily representative of the shock speed (owing to projection effects, or if the shock is propagating in already moving material).

From Fig. 4 and Table 5, we draw the following conclusions:

- For the HV blueshifted component, the wide velocity range (71 $\mathrm{km} \mathrm{s}^{-1}$ : see Table 4) implies that comparisons with our models are possible only if the shock is propagating in already moving material. In this case, the agreement with the observations is somewhat better than for scenario 1, Sect. 5.1, because the $\mathrm{OH}$ and $\mathrm{CO}$ integrated intensities are slightly lower, and because the blue wing of the $2514 \mathrm{GHz}$ $\mathrm{OH}$ line can now be fitted by the models. A J-type shock with $n_{\mathrm{H}}=10^{5} \mathrm{~cm}^{-3}, b=0.1$, and $v_{\mathrm{s}}=30 \mathrm{~km} \mathrm{~s}^{-1}$ is almost a good fit to the observations.

- For the LV blueshifted and redshifted components, the integrated intensities in the various observed lines have similar values, and their velocity widths are similar. Comparisons with shock models show that, for an inclination angle in the range of $70-75^{\circ}$, which transforms a speed of $8 \mathrm{~km} \mathrm{~s}^{-1}$ along the line of sight into a propagation speed of $25-30 \mathrm{~km} \mathrm{~s}^{-1}$, the observations can be reproduced, as may be seen in Fig. 4. In this case, J-type shocks with $n_{\mathrm{H}}=10^{5} \mathrm{~cm}^{-3}, b=0.1$, and 
$v_{\mathrm{s}}=25-30 \mathrm{~km} \mathrm{~s}^{-1}$ provide the best fit to the observations. However, the high value assumed for the inclination angle introduces additional uncertainty (see Fig. B.1).

- For the HV redshifted component, a J-type shock with $n_{\mathrm{H}}=$ $10^{5} \mathrm{~cm}^{-3}, b=0.1$, and $v_{\mathrm{s}}=30 \mathrm{~km} \mathrm{~s}^{-1}$ fits the observed emission of both $\mathrm{CO}$ and $\mathrm{OH}$.

In all these cases, the J-type solution could be combined with a less dense C-type solution (with $n_{\mathrm{H}}$ in the range $\left[10^{4}-10^{5}\right] \mathrm{cm}^{-3}$ and $v_{\mathrm{s}} \gtrsim 30 \mathrm{~km} \mathrm{~s}^{-1}$ ) without significantly detracting from the quality of the fits to the observations, as was found in Sect. 5.1.

\section{Concluding remarks}

We have reported SOFIA observations of $\mathrm{CO}$ and $\mathrm{OH}$ spectral lines in the Cep A massive SFR. We considered two approaches in our analysis of the data, based on the CO study of Cunningham et al. (2009). In the first approach, we tried to fit one shock model per blueshifted and redshifted gas component. We found that no single model from the grid of Flower $\&$ Pineau des Forêts (2015) could adequately fit these measurements. This conclusion is at variance with the findings from studies of shocks associated with low-mass star formation. For instance, Karska et al. (2014b) have shown that C- and J-type models are generally capable of fitting the emission lines of various species, observed around numerous YSOs in the Perseus cloud. Leurini et al. $(2013,2014)$ have shown that, in the massive SFR IRAS 17233-3606, $\mathrm{H}_{2} \mathrm{O}$ and $\mathrm{SiO}$ observations could be interpreted in terms of C-type shocks with a high pre-shock density $\left(n_{\mathrm{H}} \approx 10^{6} \mathrm{~cm}^{-3}\right)$ and shock velocity $\left(v_{\mathrm{s}} \approx 30 \mathrm{~km} \mathrm{~s}^{-1}\right)$. In the second approach, we assumed the existence of two outflows in the region or the coexistence of one outflow (associated with the high-velocity emission) with large-scale, bipolar cavity walls (associated to the low-velocity emission). We thus divided the lines into four velocity components and tried to fit shock models to each of them. We could fit the redshifted HV component successfully by a J-type shock with $n_{\mathrm{H}}=10^{5} \mathrm{~cm}^{-3}, b=0.1$, and $v_{\mathrm{s}}=30 \mathrm{~km} \mathrm{~s}^{-1}$. This model could be combined with a less dense C-type solution without significantly altering the comparison with the observations. This emission could arise in a young, non-stationary shock. Alternatively, it might originate in both the jet (J-type contribution) and either the bow shock or the outflow cavity walls (C-type contribution).

The present study confirms the necessity of recourse to high pre-shock densities when fitting molecular emission lines from massive SFR. A potentially significant limitation of our current models is that they exclude the effects of a UV radiation field, which might be emitted by the driving source or produced by the shock itself, and it is clear that progress will depend on further development of the shock models. We believe the observation of velocity-resolved $\mathrm{OH}$ spectra will prove to be a useful tool complementary to spectroscopic observations of $\mathrm{H}_{2} \mathrm{O}$ and $\mathrm{OI}$, e.g. at $63 \mu \mathrm{m}$ by SOFIA/GREAT - when seeking to understand the water chemistry in high-mass SFRs.

Acknowledgements. We thank an anonymous referee and M. Walmsley for comments that helped to improve this paper. We thank the SOFIA operations and the GREAT instrument teams, whose support has been essential for the GREAT accomplishments, and the DSI telescope engineering team. Based (in part) on observations made with the NASA/DLR Stratospheric Observatory for Infrared Astronomy. SOFIA Science Mission Operations are conducted jointly by the Universities Space Research Association, Inc., under NASA contract NAS297001, and the Deutsches SOFIA Institut, under DLR contract 50 OK 0901. We thank N. Cunningham for providing us with the spectacular image of the region. This work was partly funded by grant ANR-09- BLAN-0231-01 from the French Agence Nationale de la Recherche as part of the SCHISM project. It was also partly supported by the CNRS programme "Physique et Chimie du Milieu Interstellaire".

\section{References}

Anderl, S., Guillet, V., Pineau des Forêts, G., \& Flower, D. R. 2013, A\&A, 556, A69

Arce, H. G., Shepherd, D., Gueth, F., et al. 2007, Protostars and Planets V, 245

Bachiller, R., Pérez Gutiérrez, M., Kumar, M. S. N., \& Tafalla, M. 2001, A\&A, 372, 899

Bartkiewicz, A., Szymczak, M., Cohen, R. J., \& Richards, A. M. S. 2005, MNRAS, 361, 623

Benedettini, M., Busquet, G., Lefloch, B., et al. 2012, A\&A, 539, L3

Codella, C., Cabrit, S., Gueth, F., et al. 2014, A\&A, 568, L5

Cohen, R. J., Rowland, P. R., \& Blair, M. M. 1984, MNRAS, 210, 425

Cunningham, N. J., Moeckel, N., \& Bally, J. 2009, ApJ, 692, 943

Dionatos, O., Jørgensen, J. K., Green, J. D., et al. 2013, A\&A, 558, A88

Flower, D. R., \& Pineau des Forêts, G. 2003, MNRAS, 343, 390

Flower, D. R., \& Pineau des Forêts, G. 2013, MNRAS, 436, 2143

Flower, D. R., \& Pineau des Forêts, G. 2015, A\&A, 578, A63

Flower, D. R., Le Bourlot, J., Pineau des Forêts, G., \& Cabrit, S. 2003, MNRAS, 341,70

Frank, A., Ray, T. P., Cabrit, S., et al. 2014, Protostars and Planets VI, 451

Garay, G., Ramirez, S., Rodriguez, L. F., Curiel, S., \& Torrelles, J. M. 1996, ApJ, 459, 193

Gómez, J. F., Sargent, A. I., Torrelles, J. M., et al. 1999, ApJ, 514, 287

Green, J. D., Evans, II, N. J., Jørgensen, J. K., et al. 2013, ApJ, 770, 123

Guan, X., Stutzki, J., Graf, U. U., et al. 2012, A\&A, 542, L4

Guillet, V., Jones, A. P., \& Pineau Des Forêts, G. 2009, A\&A, 497, 145

Guillet, V., Pineau Des Forêts, G., \& Jones, A. P. 2011, A\&A, 527, A123

Gusdorf, A., Cabrit, S., Flower, D. R., \& Pineau Des Forêts, G. 2008, A\&A, 482, 809

Gusdorf, A., Anderl, S., Güsten, R., et al. 2012, A\&A, 542, L19

Gusdorf, A., Giannini, T., Flower, D. R., et al. 2011, A\&A, 532, A53

Gyulbudaghian, A. L., Glushkov, Y. I., \& Denisyuk, E. K. 1978, ApJ, 224 L137

Heyminck, S., Graf, U. U., Güsten, R., et al. 2012, A\&A, 542, L1

Hodapp, K.-W. 1994, ApJS, 94, 615

Hollenbach, D., \& McKee, C. F. 1989, ApJ, 342, 306

Hughes, V. A., \& Wouterloot, J. G. A. 1982, A\&A, 106, 171

Hughes, V. A., \& Wouterloot, J. G. A. 1984, ApJ, 276, 204

Karska, A., Herczeg, G. J., van Dishoeck, E. F., et al. 2013, A\&A, 552, A141

Karska, A., Herpin, F., Bruderer, S., et al. 2014a, A\&A, 562, A45

Karska, A., Kristensen, L. E., van Dishoeck, E. F., et al. 2014b, A\&A, 572, A9

Klein, B., Hochgürtel, S., Krämer, I., et al. 2012, A\&A, 542, L3

Kristensen, L. E., van Dishoeck, E. F., Benz, A. O., et al. 2013, A\&A, 557, A23

Le Bourlot, J., Pineau des Forêts, G., Flower, D. R., \& Cabrit, S. 2002, MNRAS, 332, 985

Lesaffre, P., Pineau des Forêts, G., Godard, B., et al. 2013, A\&A, 550, A106

Leurini, S., Codella, C., Gusdorf, A., et al. 2013, A\&A, 554, A35

Leurini, S., Gusdorf, A., Wyrowski, F., et al. 2014, A\&A, 564, L11

Lindberg, J. E., Jørgensen, J. K., Green, J. D., et al. 2014, A\&A, 565, A29

Moscadelli, L., Reid, M. J., Menten, K. M., et al. 2009, ApJ, 693, 406

Müller, H. S. P., Thorwirth, S., Roth, D. A., \& Winnewisser, G. 2001, A\&A, 370, L49

Müller, H. S. P., Schlöder, F., Stutzki, J., \& Winnewisser, G. 2005, J. Mol. Struct., 742,215

Narayanan, G., \& Walker, C. K. 1996, ApJ, 466, 844

Pickett, H. M., Poynter, R. L., Cohen, E. A., et al. 1998, J. Quant. Spectr. Rad. Transf., 60, 883

Podio, L., Codella, C., Gueth, F., et al. 2015, A\&A, 581, A85

Pravdo, S. H., Tsuboi, Y., Uzawa, A., \& Ezoe, Y. 2009, ApJ, 704, 1495

Rodriguez, L. F., Moran, J. M., \& Ho, P. T. P. 1980, ApJ, 240, L149

Rodriguez, L. F., Garay, G., Curiel, S., et al. 1994, ApJ, 430, L65

Sargent, A. I. 1977, ApJ, 218, 736

Snell, R. L., Loren, R. B., \& Plambeck, R. L. 1980, ApJ, 239, L17

Tan, J. C., Beltrán, M. T., Caselli, P., et al. 2014, Protostars and Planets VI, 149

Tappe, A., Lada, C. J., Black, J. H., \& Muench, A. A. 2008, ApJ, 680, L117

Tappe, A., Forbrich, J., Martín, S., Yuan, Y., \& Lada, C. J. 2012, ApJ, 751, 9

van Dishoeck, E. F., Kristensen, L. E., Benz, A. O., et al. 2011, PASP, 123 138

van Dishoeck, E. F., Herbst, E., \& Neufeld, D. A. 2013, Chem. Rev., 113, 9043 van Dishoeck, E. F., Bergin, E. A., Lis, D. C., \& Lunine, J. I. 2014, in Protostars and Planets VI, eds. H. Beuther, R. S. Klessen, C. P. Dullemond, \& T. Henning (Tucson: University of Arizona Press), 835

Vlemmings, W. H. T. 2008, A\&A, 484, 773

Wampfler, S. F., Herczeg, G. J., Bruderer, S., et al. 2010, A\&A, 521, L36 Wampfler, S. F., Bruderer, S., Kristensen, L. E., et al. 2011, A\&A, 531, L16 Wampfler, S. F., Bruderer, S., Karska, A., et al. 2013, A\&A, 552, A56 Wright, C. M., Drapatz, S., Timmermann, R., et al. 1996, A\&A, 315, L301 


\section{Appendix A: Triple-Gaussian fits of the $\mathrm{OH}$ $1835 \mathrm{GHz}$ and $\mathrm{CO}(16-15)$ lines}

This Appendix shows our Gaussian decomposition of the $\mathrm{OH}$ $1835 \mathrm{GHz}$ and $\mathrm{CO}(16-15)$ emission lines in Figs. A.1 and A.2.

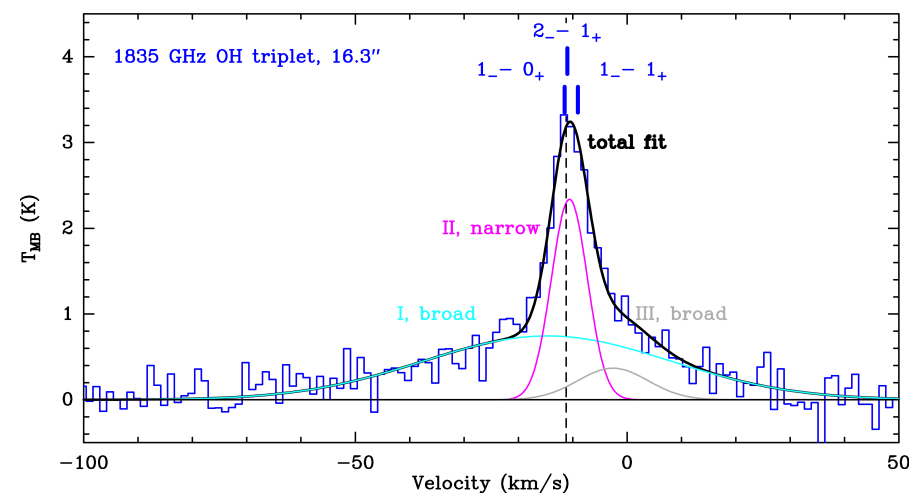

Fig. A.1. Triple-Gaussian fit of the $1835 \mathrm{GHz} \mathrm{OH}$ triplet (blue line, from Fig. 2): blueshifted (light blue line), ambient (pink line), redshifted (grey line) components, and total fit (black line). The vertical dashed line is at the cloud $v_{\mathrm{lsr}}$ of $-11.2 \mathrm{~km} \mathrm{~s}^{-1}$ (Narayanan \& Walker 1996; Gómez et al. 1999).

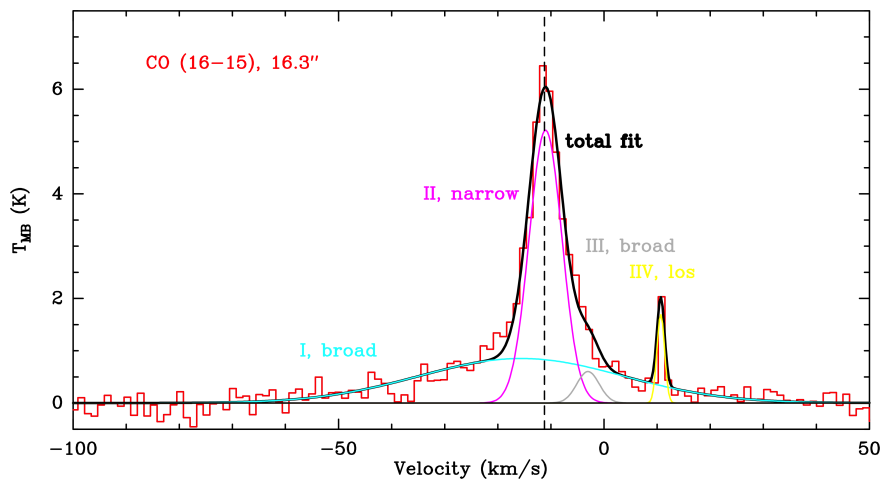

Fig. A.2. Triple-Gaussian fit of the $\mathrm{CO}(16-15)$ transition (red line, from Fig. 2): blueshifted (light blue line), ambient (pink line), redshifted (grey line) components, and total fit (black line). An additional component has been fitted with respect to the $\mathrm{OH}$ line in Fig. A.1: a narrow $\mathrm{CO}$ feature at $10.7 \mathrm{~km} \mathrm{~s}^{-1}$ due to mesospheric $\mathrm{CO}$ over-compensated by the correction for the atmospheric opacity and well reproduced by a fourth Gaussian (yellow line). The vertical dashed line is at the cloud $v_{\text {lsr }}$ of $-11.2 \mathrm{~km} \mathrm{~s}^{-1}$ (Narayanan \& Walker 1996; Gómez et al. 1999).

\section{Appendix B: Integrated intensities at arbitrary inclinations}

In this Appendix, we briefly discuss the influence of inclination angle on the integrated intensity of the $\mathrm{OH} 1835 \mathrm{GHz}$ line, thereby illustrating the difficulties encountered when comparing shock models with observations of $\mathrm{OH}$ emission lines in massive star-forming environments such as Cep A. We base our discussion on two particular shock models: a J-type model with $n_{\mathrm{H}}=10^{5} \mathrm{~cm}^{-3}, b=0.1$, and $v_{\mathrm{s}}=30 \mathrm{~km} \mathrm{~s}^{-1}$ and a C-type model with $n_{\mathrm{H}}=10^{6} \mathrm{~cm}^{-3}, b=1$, and $v_{\mathrm{s}}=30 \mathrm{~km} \mathrm{~s}^{-1}$. These models simulate the observations most closely (see Fig. 4). For these two cases, we show the variation in the neutral temperature and optical depth of the line considered through the shock
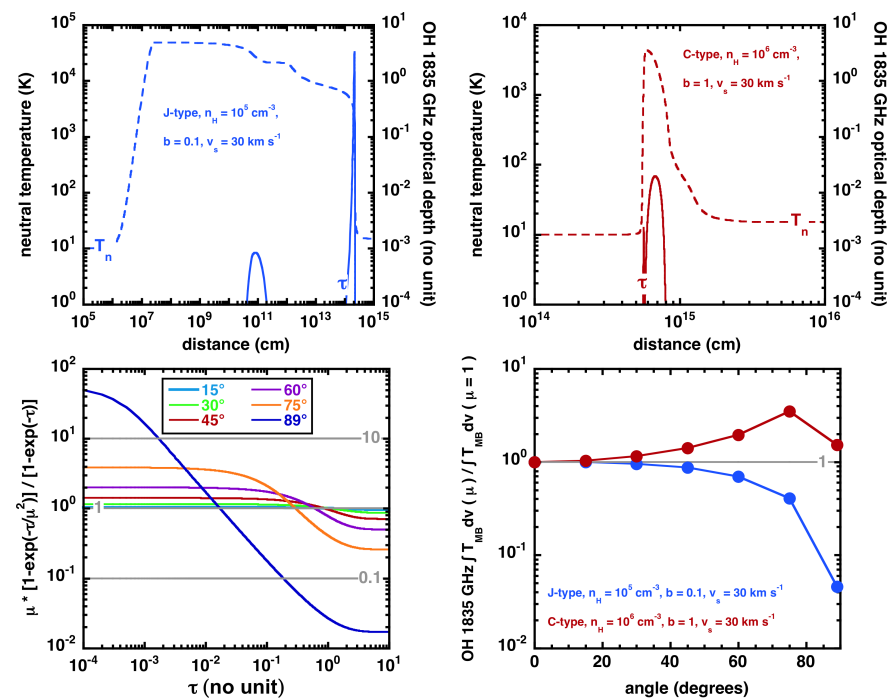

Fig. B.1. Upper panels: evolution of the neutral temperature and optical depth of the $\mathrm{OH}$ line at $1835 \mathrm{GHz}$ along the shock layer (versus a distance parameter along the direction of propagation of the shock) for a J-type model with $n_{\mathrm{H}}=10^{5} \mathrm{~cm}^{-3}, b=0.1$, and $v_{\mathrm{s}}=30 \mathrm{~km} \mathrm{~s}^{-1}$ (left) and a C-type model with $n_{\mathrm{H}}=10^{6} \mathrm{~cm}^{-3}, b=1$, and $v_{\mathrm{s}}=30 \mathrm{~km} \mathrm{~s}^{-1}$ (right). Lower left panel: variation in the factor $\mu \cdot\left(1-\mathrm{e}^{-\tau / \mu^{2}}\right) /\left(1-\mathrm{e}^{-\tau}\right)$ with the optical depth for six inclination angles. Lower right panel: variation in the ratio $\left[\int T \mathrm{~d} v(\mu)\right] /\left[\int T \mathrm{~d} v(\mu=1)\right]$ with the inclination angle for the two shock models considered.

layer (versus a distance parameter along the direction of propagation of the shock) in the upper panels of Fig. B.1. The line is optically thick in the region of the J-type shock, while it remains constantly optically thin in the C-type shock.

We consider an arbitrary inclination angle, $\theta$, and define $\mu=\cos \theta$. Then, at each point in the shock layer, the line temperature is effectively multiplied by a factor $\left(1-\mathrm{e}^{-\tau / \mu^{2}}\right) /\left(1-\mathrm{e}^{-\tau}\right)$ (formula A.16 of Gusdorf et al. 2008), where $\tau$ is the so-called LVG optical depth (see formula A.2 of Gusdorf et al. 2008). Simultaneously, the velocity of the layer is multiplied by a projection factor along the photon path, $\mu$. In effect, the integrated intensity evaluated at each point of the shock should be multiplied by a factor $\mu \cdot\left(1-\mathrm{e}^{-\tau / \mu^{2}}\right) /\left(1-\mathrm{e}^{-\tau}\right)$. We display the variation in this factor with the optical depth for six inclination angles $\left(15,30,45,60\right.$, and $\left.89^{\circ}\right)$ in the lower left-hand panel of Fig. B.1. This figure shows that the correction factor is less than 1 for optically thick transitions and greater than 1 for optically thin transitions, as might have been anticipated. Because the correction factor is applied point-by-point to the integrated intensity and depends on the inclination angle, we have not attempted to extend this study to all models of the grid.

The variation in the ratio $\left[\int T \mathrm{~d} v(\mu)\right] /\left[\int T \mathrm{~d} v(\mu=1)\right]$ with the inclination angle is shown in the lower right-hand panel of Fig. B.1 for both shock models. In the J-type model, one can see that the integrated intensity is reduced by the correction for the inclination angle, regardless of its value, because the line is optically thick in the post-shock region, where the emission is most significant. In the C-type model, the integrated intensity is increased by the correction for the inclination angle (with a maximum correction factor of $\sim 3.5$ for an angle of $75^{\circ}$ ), because the line is always optically thin. However, this correction is insufficient to fully reconcile the integrated intensity with the observed value in the framework of the discussion in Sects. 5.1 or 5.2. 